\title{
Cullivo de Trigo harinero 'Triticum aestivum, una alternativa para la soberanía nutricional y adaptación ante el cambio climático, en el departamento de Jinotega
}

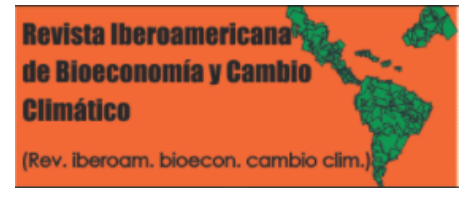

\section{Cultivation of bread wheat Triticum aestivum, an alternative for nutritional sovereignty and adaptation to climate change, in the department of Jinotega}

\author{
Estrada Santana, David Concepción; Zuniga González, Carlos \\ Alberto; Hernández Rueda, Mauricio José; Marinero Orantes, Edgard \\ Antonio; Editor Academico Dr. Angel sol Sanchez
}

David Concepción Estrada Santana david.estrada@post.unanleon.edu.ni Universidad Nacional Autónoma de Nicaragua. UNAN-León., Nicaragua

iD Carlos Alberto Zuniga González czuniga@ct.unanleon.edu.ni

Universidad Nacional Autónoma de Nicaragua. UNAN-León. Centro de Investigación en Ciencias Agrarias. Escuela Ciencias Agrarias y Veterinaria. Departamento de Agroecología., Nicaragua

(iD) Mauricio José Hernández Rueda mauriciohernandez5@gmail.com

Universidad Nacional Autónoma de Nicaragua.

UNAN-León. Centro de Investigación en Ciencias Agrarias. Escuela Ciencias Agrarias y Veterinaria. Departamento de Agroecología., Nicaragua

iD Edgard Antonio Marinero Orantes edgar.marinero@ues.edu.sv

Universidad de El Salvador, Miembro del Consejo de Investigaciones Científicas de la Universidad de El Salvador (CIC-UES)., El Salvador

Editor Academico Dr. Angel sol Sanchez

Colegio de postgraduados, Mexico, Mexico

Revista Iberoamericana de Bioeconomía y Cambio Climático

Universidad Nacional Autónoma de Nicaragua, León, Nicaragua ISSN-e: 2410-7980

Periodicidad: Semestral

vol. 2, núm. 1, 2016

czuniga@ct.unanleon.edu.ni

Recepción: 02 Febrero 2015

Aprobación: 26 Marzo 2016
Resumen: El presente ensayo, se centró en dar a conocer la importancia agronómica y económica del rubro del trigo harinero Triticum aestivum, como estrategia de Soberanía, Seguridad nutricional local y de Desarrollo Rural en tres zonas climáticamente diferentes del departamento de Jinotega, debido a su alto valor nutricional de proteínas $10.1 \%$, carbohidratos $76.7 \%$ y fibras $47.55 \%$, que aporta este rubro a las familias productoras del mundo. Así incluirlo en los programas de gobierno locales para contra restar la pobreza extrema de Nicaragua; y disminuir las importaciones económica de 36.1 millones de dólares que invierte Nicaragua anualmente. Por otro lado, incorporar el trigo harinero en los proyectos de sistemas de producción agrícolas diversificado con enfoque agroecológico para lograr la adaptabilidad ante los impactos del cambio climático.

Palabras clave: Trigo, Diversificación, Comodity financiero, Cambio climático, Soberanía y Seguridad Alimentaria Nutricional.

Abstract: This essay focused on making known the agronomic and economic importance of the bread wheat item Triticum aestivum, as a strategy of Sovereignty, local nutritional security and Rural Development in three different climatic zones of the department of Jinotega, due to its high value nutritional protein $10.1 \%$, carbohydrates $76.7 \%$ and fibers $47.55 \%$, which this item contributes to the world's producer families. Thus, include it in local government programs to counteract extreme poverty in Nicaragua; and reduce the economic imports of 36.1 million dollars that Nicaragua invests annually. On the other hand, incorporate bread wheat in projects of diversified agricultural production systems with an agroecological approach to achieve adaptability to the impacts of climate change.

Keywords: Wheat, Diversification, Financial comfort, Climate change, Food Sovereignty and Nutritional Security. 
DOI: https://doi.org/10.5377/ribcc.v2i1.5705

Autor de correspondencia: david.estrada@post.unanleon.edu.ni

\section{INTRODUCCIÓN}

El trigo (Triticum aestivum) tiene sus orígenes en la antigua Mesopotamia específicamente en Asia menor, Asia Central y África, es uno de los tres cereales más producidos globalmente, junto al maíz y el arroz, y el más ampliamente consumido por el hombre en la civilización occidental desde la antigüedad. El grano del trigo es utilizado para hacer harina, harina integral, sémola, cerveza y una gran variedad de productos alimenticios. (INFOAGRO, 2013).

Según Altieri y Toledo (2011), la amenaza a la seguridad alimentaria, es el resultado directo del modelo agroindustrial que se caracteriza por los monocultivos a gran escala y los cultivos transgénicos, mientras los agros combustibles ejercen mayor presión sobre los ecosistemas degradados, socavando con ello aún más la capacidad de éstos para el suministro de alimentos, fibra y energía a una población humana en crecimiento.

La propiedad más importante del trigo es la capacidad de cocción de la harina debida a la elasticidad del gluten que contiene. Esta característica permite la panificación, constituyendo un alimento básico para el hombre. El trigo se cultiva en todo el mundo siendo la principal área de cultivo la zona templada del hemisferio norte, (Cañarejo, P; Adolfo, S, 2013).

En cuanto a sus características de adaptabilidad para un buen desarrollo; se consigue en terreno cargado de marga y arcilla, aunque el rendimiento es satisfactorio en terrenos más ligeros. Prospera en climas subtropicales, moderadamente templados y fríos. El trigo se puede dividir en tres grandes grupos: trigos duros (hard) o panificables, trigos suaves (soft) o galleteros y trigos cristalinos (durum) o semoleros y pasteleros. (Financiera rural, 2010).

En Nicaragua el cultivo de trigo es poco conocido, entre las pocas experiencias que se tiene de su establecimiento en el país se reporta aproximadamente hace casi 34 años, con el apoyo de la empresa Gemina que realizó siembras de trigo cerca de la comunidad de Teotecacinte, en el Valle de Jalapa, de lo cual logró recolectar alrededor de 39 quintales de semilla de trigo por manzana. Ese experimento comenzó en 1978, cuando se pidió semilla de trigo de la variedad Pénjamo al Centro Internacional de Mejoramiento del Maíz y del trigo (CIMMYT), de México, con lo cual logró realizar una siembra comercial en 35 manzanas. El trigo recolectado fue llevado por Gemina a sus planteles en Chinandega y se sacó una harina intermedia de alta calidad, pero el experimento se canceló (Villaseñor, O, et al, 2009).

A partir de lo antes planteado, el objetivo del estudio es la implementación de un sistema de producción agroecológico utilizando variedades criollas como el trigo harinero variedad Jupateco, quien representa una oportunidad para demostrar la importancia económica y agronómica de este cultivo, en la seguridad alimentaria local bajo dos sistema de siembra al voleo y en surcos en tres zonas climáticamente diferentes de los municipios de La Concordia, Pantasma y Jinotega como estrategia de adaptabilidad ante el cambio climático. Así incorporara a los productores dedicados a este rubro a que puedan acceder a nuevos mercados que permitan mejorar su calidad de vida y así poder lograr un desarrollo rural territorial.

NotAS DE AUTOR 


\section{REVISIÓN DE LA LiTERATURA}

\section{Formulación de un sistema de producción agroecológica de trigo harinero basado en la seguridad alimentaria y nutricional, y adaptación ante el cambio climático}

La estrategia más eficaz para hacer adelantos constantes y sostenibles en los Objetivos de Desarrollo Sostenible es tratar todos los objetivos de forma integrada. Sin embargo, para lograr el éxito sobre el terreno, se necesita un paquete de tecnologías y servicios bien definidos para cada objetivo. ${ }^{* *} 1 \mathrm{El}$ Task Force on Hunger está proveyendo orientación adecuada para elaborar estos paquetes en caso de hambruna. Esta labor es de especial relevancia, puesto que, si cada objetivo se persigue de manera separada, sin tomar en cuenta su vínculo con los demás objetivos, el complejo proceso del desarrollo humano y económico se reduce a una serie de intervenciones fragmentadas, contradictorias e insostenibles. En consecuencia, lo más indicado es un enfoque global y armonioso de desarrollo. IFPRI (2004). Por tanto, el desarrollo de un modelo productivo agrícola sostenible apostaría a reducir el objetivo de desarrollo sostenible número 1 . $^{* * *}$

** El Task Force on Hunger (ente de asesoramiento en lo concerniente al logro del objetivo de reducir el hambre a la mitad para 2015 .

*** Objetivo de desarrollo sostenible 1. Erradicación de la pobreza extrema.

Requiere una humedad relativa entre 40 y $70 \%$; desde el espigamiento hasta la cosecha es la época que tiene mayores requerimientos en este aspecto, ya que exige una humedad relativa entre el 50 y $60 \%$ y un clima seco para su maduración (Made-in- argentina, 2010).

El cultivo de trigo tiene unos bajos requerimientos de agua, ya que se puede cultivar en zonas donde caen precipitaciones entre 25 y $2800 \mathrm{~mm}$ anuales de agua, aunque un $75 \%$ del trigo crece entre los 375 y $800 \mathrm{~mm}$. La cantidad optima es de 400-500 mm/ciclo.

Los mejores suelos para su crecimiento deben ser sueltos, profundos, fértiles y libres de inundaciones, y deben tener un pH entre 6,0 y 7,5; en terrenos muy ácidos es difícil lograr un adecuado crecimiento (Made-in-argentina, 2010).

La amnesia de los investigadores nicaragüenses y el error de la traducción de Sorghum bicolor dándole el nombre de trigo (Triticum aestivum), por parte del doctor Jaime Incer Barquero en el año1975 de los escritos en inglés por Thomas Belt en su libro El Naturalista en Nicaragua, (según Frenzel, 2014), permitió difundir que no existen en Nicaragua condiciones agro climática para la producción de trigo harinero en las aulas de las universidades estatales y privadas que promueven la educación en agricultura dentro y fuera del país.

Estudios realizados por Iglesias, et al 2012-2013 en la producción de trigo harinero de la variedad Jupateco, demuestra que, si existen condiciones agroecológicas propicias de temperatura que van desde los 24 a los 29 grados Celsius, humedades relativas del 75 al $85 \%$ y precipitaciones de 800 a $1500 \mathrm{~mm}$ anuales para la producción de trigo harinero en la zona de la Colmena abajo del municipio de la concordia. Así mismo demuestra que hay más de 8000 manzanas ociosas de tierra donde se puede cultivar trigo harinero.

2.2 Sistema de producción más utilizado del cultivo de trigo.

La humanidad a través de su historia ha sembrado trigo siguiendo el método tradicional (siembra semejante al voleo) y los incrementos en producción, hasta ahora logrados, han sido fundamentalmente debidos al mejoramiento de los genotipos, en su habilidad para explorar el medio ambiente particular propiciado por este método de siembra.

Estos incrementos se mejoraron con el uso de insumos empleados en la producción moderna de trigo. De esta manera los científicos que se han dedicado a mejorar la producción de este cereal han respetado la metodología para sembrar trigo y la aceptan como la más idónea. (Miller, 2000).

Hoy en día la tecnología disponible para el productor en la mayoría de los países de latino america entre ellos México, consiste en variedades mejoradas de alto rendimiento y en el uso de dosificaciones altas de insumos de producción, fundamentalmente, altas cantidades de semilla, fertilizante, agua y agroquímicos en general; tecnología que no ha considerado las interacciones de tipo complejo, principalmente, con los regímenes de malezas. Por otra parte, además de dejar poco margen de ganancias, no considera el deterioro del ambiente, al cual contribuye al usar dosificaciones excesivas de algunos factores modificables de la producción (pesticidas y fertilizantes). (Moreno, 2011).

Como consecuencia del uso continuo de esta tecnología de producción de trigo, las poblaciones de malezas y enfermedades se han incrementado y generalizado en los campos agrícolas. Esta situación ha forzado a muchos de los productores a cambiar temporalmente de cultivo, es decir, a la siembra de cártamo o algodón, que son cultivos de escarda, y permiten un control más eficiente de malezas. El uso de agroquímicos (fertilizantes, insecticidas, fungicidas y herbicidas) en trigo, ha sido una práctica común para el productor; sin embargo, dichos herbicidas por su forma de acción incrementan los problemas contaminantes ambientales en el mundo acelerando aún más el calentamiento global de la tierra. (Moreno, 2011). 
Por otro lado, la pérdida de la diversidad biológica de muchos cultivos agrícolas se debe a la dependencia de paquetes tecnológicos de semillas mejoradas ofertadas por las trasnacionales que dominan la agroindustria. Semillas que no tienen el potencial de garantizar la seguridad y soberanía alimentaria de los campesinos ya que dependen de una serie de insumos sin los cuales no funcionan y son altamente vulnerables al cambio climático 21 (Watson,2012, (Platero G, et al, 2015).

El cambio del sistema de siembra como es en surco ha demostrado maximizar y proteger el suelo manteniendo los niveles de producción estable y en su mayoría disminuye los costos de producción. (Moreno, 2014)

El enfoque convencional de la agricultura ha producido aumentos importantes en la productividad agropecuaria y ha logrado una cobertura significativa en la oferta de alimentos. Sin embargo, a pesar de estos logros, este modelo viene afectando el ambiente, especialmente los recursos naturales como el bosque, el suelo, el agua y la biodiversidad de plantas y animales. En las últimas dos décadas han surgido diferentes teorías y propuestas encaminadas a buscar una mejor armonía entre la agricultura y el ambiente, sobresaliendo como enfoque principal la Agroecología, la cual muestra como unidad principal la optimización del agroecosistema. (Restrepo, J., et al, 2000).

La implementación de un modelo producción agroecológico del cultivo de trigo utilizando el sistema de siembra de surco y la aplicación de micro organismo como micorrizas, trichoderma e insectos benéficos como crisoperla y trichogramma puede ser el camino para lograr la sostenibilidad ambiental y de producción del trigo, en el centro norte del Nicaragua, donde existen condiciones agroclimáticas para el desarrollo del trigo y suplir las necesidades de desnutrición e inseguridad nutricional que enfrentan las comunidades rurales de los municipios de la Concordia, Pantasma y Jinotega, especial atención a niños, adolescentes embarazadas y personas de la tercera edad.

Para ello requiere un compromiso de gobierno que garantice la aplicación de la ley 7652 a todos los niveles en especial a los pequeños y medianos productores, quienes son los mayores aportadores a la economía del país, así estaría respaldando la ley 6933 cumpliendo con el mandato de la constitución en su Arto. 63. Es derecho de los nicaragüenses estar protegidos contra el hambre. El estado promoverá programas que aseguren una adecuada disponibilidad de alimentos y una distribución equitativa de los mismos.

Así mismo al producir trigo harinero con enfoque agroecológico, se conservará y preservará el bien tierra, agua y demás recursos naturales, así como preservación de la única variedad de trigo Jupateco que ha quedado en tierras nicaragüenses, en donde México ya desde de más de una década la perdió al sacar un híbrido de esta variedad. México, era el único lugar donde estaba el germoplasma de trigo Jupateco, Moreno (2011). Por tanto, Nicaragua trasciende a tener la única variedad del mundo, al tenerse por más de 40 años se tiene como una variedad acriollada.

3 El trigo, otro commodity financiarizado.

Como todas las materias primas su precio reacciona a factores de oferta y demanda; pero, a diferencia del resto, las condiciones climatológicas juegan un papel todavía más crucial en la calidad harinera.

Aunque en el futuro se llegan a negociar desde la India (In) hasta Argentina y Hungría, la atención mundial está en los mercados estadounidenses: Bolsa de Comercio de Kansas City (KCBOT, en inglés), Bolsa de Granos de Minneapolis (MGE, en inglés) y, más aún, Bolsa de comercio de Chicago (CBOT en inglés). (Oscar Ugarteche. L., David Segovia Villeda, 2010).

Este gráfico 1. Se muestra la tendencia reciente en el precio futuro del trigo comerciado en el CBOT: a) alza impresionante del $434 \%$ desde $01 / 2000$ hasta 02/2008 y b) desaceleración del 51\% en 12/2014 a partir del máximo alcanzado en 02/2008. Destaca que en comparación con el resto de las materias primas, el boom terminó en $2008 \mathrm{y}$, de mayor importancia, el contraste entre el crecimiento extraordinario en un periodo tan corto de tiempo como el 08/2005-02/2008 y la caída pausada (que titubeo en $05 / 2010$ y 02/2012) en los 7 años posteriores. Tan alarmante fluctuación responde únicamente a los factores demográficos, económicos, naturales y geopolíticos que determinan la oferta y demanda. 


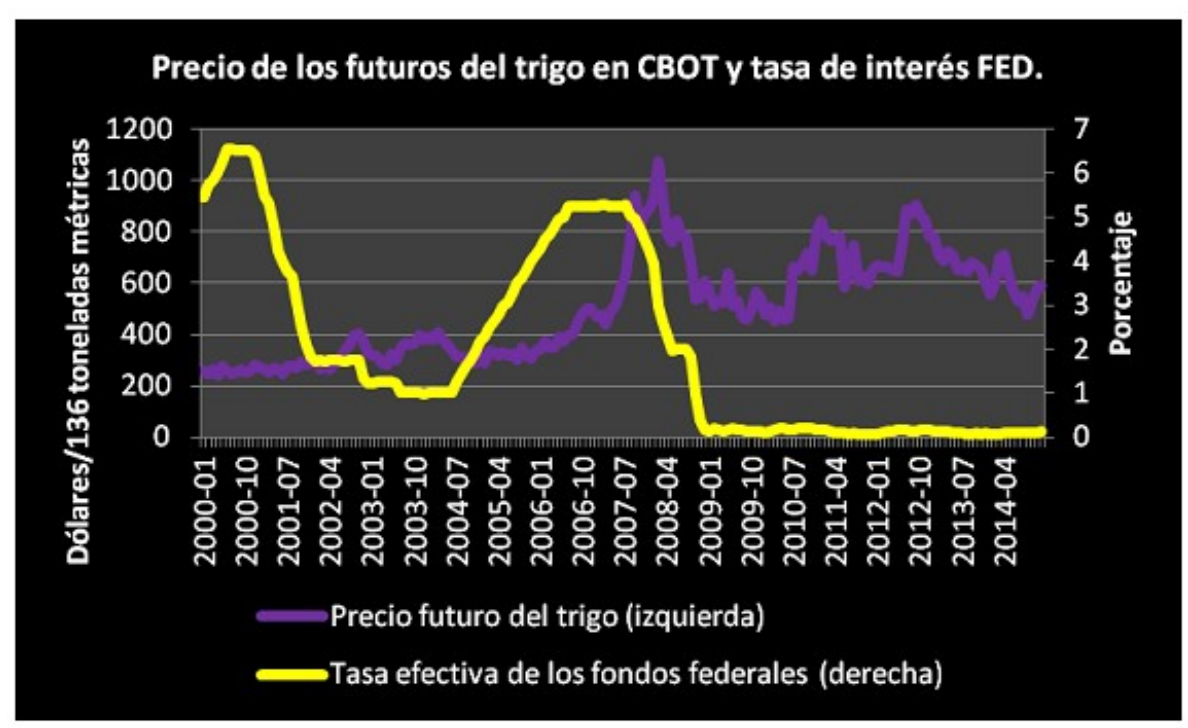

GRAFICO 1

Precio de los futuros del trigo en el CBOT y la tasa de interés del FED

Oscar Ugarteche. L., David Segovia Villeda

En Nicaragua a pesar de que el cultivo de trigo es un rubro olvidado y muchos menos se producen, en el año 2012 se comercializó el quintal de trigo a 900 córdobas como grano y 1200 córdobas como semilla en los mercados de Estelí y Jinotega. Siendo financiado la producción obtenida en ese año por organismos de investigación nacionales e internacionales como Swissaid. Al despertar interés en la producción y comercialización en Nicaragua existen financiadores como Banco produzcamos, Banpro y financieras locales como pro credit y Fondo de Desarrollo Local (FDL).(Joachim von Braun, 2003)

La Unión Europea (UE), China (Ch), India (In), Estados Unidos (EU) y Rusia (Ru) son los principales consumidores $(\mathrm{Md})$ y productores $(\mathrm{Ms})$ mundiales del grano. Del lado de la demanda, la participación de la UE, Ch e In ha sobre compensado el estancamiento de Ru y EU: el crecimiento de $26 \%, 18 \%, 17 \%$ y $12 \%$ en los cuatro primeros explica el alza de 837 a 1010 en principales consumidoress. Respecto a la oferta, ahora se le suma Ru al ancla de arrastre conformada por la UE, Ch e India, ahora el incremento de productores va de 804 a 966. A pesar de que los datos de las fuerzas del mercado dan cierto aliento a los diagnósticos conservadores, la estrechez entre principales consumidores y productores bajo un escenario de gran volatilidad en el precio del trigo hace pensar en otro factor de gran envergadura. (Oscar Ugarteche. L., David Segovia Villeda, 2010).

En Nicaragua uno los productos importados mencionados en el Informe ejecutivo de Comercio Exterior entre los meses de enero a octubre del 2012 es el trigo con un total de 87,928.1 Toneladas métricas; lo que representa un gasto de 36.1 millones de dólares (MIFIC, 2012). El desarrollo productivo de esta actividad es una oportunidad para los pequeños, medianos y grandes productores dedicados a este rubro, teniendo en cuenta que el trigo harinero de variedad Jupateco demostró tener rendimientos productivos aceptables en la comunidad Colmena abajo del municipio de la Concordia y su tendencia en la comunidad es de incrementarse las áreas de siembra y dicho rubro tendría un impacto positivo en la seguridad alimentaria de las comunidades y en la generación de nuevas fuentes de ingresos económico, así como una estrategia de adaptabilidad climática y sostenibilidad para los pequeños y medianos productores. (Tabla N1) 
$\mathrm{T} 1$

Principales consumidores de trigo (millones de toneladas)

Principales consumidores de trigo (millones de toneladas)

\begin{tabular}{ccccccc}
\hline & Ch & UE & In & EU & Ru & Wd \\
\hline 2000 & 109.48 & 91.22 & 69.74 & 36.19 & 34.94 & 837.5 \\
2001 & 109.76 & 93.34 & 71.08 & 32.43 & 38.41 & 858.5 \\
2002 & 107.57 & 98.32 & 74.15 & 30.44 & 38.67 & 872.6 \\
2003 & 107.3 & 93.19 & 71.2 & 32.49 & 36 & 860.5 \\
2004 & 104.52 & 118.73 & 73.18 & 31.78 & 38.76 & 868.9 \\
2005 & 103.6 & 121.31 & 73.83 & 31.32 & 38.7 & 879.5 \\
2006 & 104.05 & 119.16 & 74.01 & 30.94 & 37.87 & 884.1 \\
2007 & 109.96 & 117,72 & 75.62 & 28.61 & 36.76 & 870.7 \\
2008 & 113.15 & 124.96 & 77.16 & 34.29 & 37.5 & 905.1 \\
2009 & 114.33 & 126.66 & 78.13 & 30.97 & 39.55 & 919.1 \\
2010 & 117.77 & 123.04 & 80.02 & 31.71 & 37.74 & 930.3 \\
2011 & 125.32 & 128.71 & 82 & 31.94 & 38.48 & 979.5 \\
2012 & 124.46 & 118.03 & 84.58 & 37.76 & 34.76 & 970.5 \\
2013 & 127.6 & 116.22 & 88 & 34.15 & 34.66 & 990.7 \\
2014 & 131.08 & 123.59 & 89.65 & 32.56 & 36.85 & 1010.6
\end{tabular}

Oscar Ugarteche. L., David Segovia Villeda

La demanda por calidad nutricional en los alimentos, impulsada por una población cada vez más consciente de lo que consume, puede ser la base para el desarrollo de negocios que aprovechen las cualidades funcionales del trigo, hasta hoy poco difundidas y durante largo tiempo subestimadas. Para el consumidor común el trigo evoca una imagen convencional, la de un cultivo con el que siempre se ha manufacturado el pan y otros alimentos de consumo cotidiano. Por otra parte, es habitual que las dietas sobre todo aquellas basadas en un bajo consumo de carbohidratos recomienden limitar el consumo de pan y otros farináceos para controlar el peso corporal. Investigaciones recientes acerca del papel que juegan en la salud tanto la fibra dietética como los antioxidantes que contienen, podrían hacer cambiar esta visión si se revaloriza el rol del trigo y sus derivados en la dieta. (Zuñiga, 2007).

\subsection{Fibra dietética del grano de trigo.}

Según Zuniga 2007, la fibra dietética, constituida por carbohidratos no digeribles y lignina presentes naturalmente en la dieta de origen vegetal, ha recibido creciente atención por los numerosos efectos saludables que acarrea su consumo habitual. Varios estudios afirman que la fibra dietética insoluble no solo aumenta el volumen de las deposiciones y regula el tránsito intestinal; también causa una disminución de la absorción de lípidos e interfiere con la circulación enterohepática de sales biliares, lo que contribuye a disminuir los niveles de colesterol.

Por otra parte, la fibra dietética soluble aumenta la viscosidad del contenido intestinal, lo que retarda la absorción de carbohidratos y contribuye a disminuir los índices glicémico e insulinémico de la dieta. También, proporciona un sustrato fermentable para la microflora intestinal, lo que estimula su crecimiento y con ello la producción de ácidos grasos de cadena corta, que son el principal sustrato para las células de la mucosa intestinal. Se estima que la suma de los efectos descritos contribuye a disminuir la probabilidad de desarrollo de ciertos tipos de cáncer, enfermedad coronaria, diabetes y obesidad, efectos beneficiosos observados en poblaciones que consumen altos niveles de fibra dietética (Sanchez, 2009). 
Los granos de los cereales son ricos en fibra dietética. Pero como ésta se localiza principalmente en la cáscara, el consumo de granos refinados, por ejemplo, harina blanca de trigo, aporta a las personas solo una fracción de la fibra presente en el grano entero (cuadro 1). En trigo predomina la fibra dietética de tipo insoluble, y sus efectos beneficiosos en la fisiología del aparato digestivo han sido demostrados por distintos estudios.

\section{CUADRO 1}

Composición tipica de macro y micronutrientes del grano de trigo y su distribución en distintos productos de molienda

\begin{tabular}{|c|c|c|c|c|c|}
\hline Nutriente & $\begin{array}{l}\text { Unidades } \\
\text { por } 100 \mathrm{~g}\end{array}$ & Grano & $\begin{array}{c}\text { Harina } \\
\text { de grano entero }\end{array}$ & Refinada & Cáscara \\
\hline \multicolumn{6}{|c|}{ Análisis proximal } \\
\hline Proteinas & $g$ & 12,6 & 13,7 & 9,7 & 15,6 \\
\hline Almidón & g & 62,4 & 60,0 & 58,9 & 14,1 \\
\hline Lipidos & g & 1,54 & 1,87 & 1,48 & 4,25 \\
\hline Cenizas & $g$ & 1,57 & 1,60 & 0,58 & 5,79 \\
\hline Fibra dietética & $g$ & 12,2 & 12,2 & 5,5 & 42,8 \\
\hline \multicolumn{6}{|l|}{ Minerales } \\
\hline Hierro & $\mathrm{mg}$ & 3,19 & 3,88 & 1,26 & 10,57 \\
\hline Magnesio & $\mathrm{mg}$ & 126 & 138 & 25 & 611 \\
\hline Fósforo & $\mathrm{mg}$ & 288 & 346 & 107 & 1.013 \\
\hline Potasio & $\mathrm{mg}$ & 363 & 405 & 149 & 1.182 \\
\hline Zinc & $\mathrm{mg}$ & 2,65 & 2,93 & 1,02 & 7,27 \\
\hline Cobre & $\mathrm{mg}$ & 0,43 & 0,38 & 0,19 & 1,00 \\
\hline Selenio & ug & 71 & 71 & 15 & 78 \\
\hline \multicolumn{6}{|l|}{ Vitaminas } \\
\hline Tiamina & $\mathrm{mg}$ & 0,38 & 0,45 & 0,19 & 0,52 \\
\hline Riboflavina & $\mathrm{mg}$ & 0,12 & 0,22 & 0,07 & 0,58 \\
\hline Niacina & $\mathrm{mg}$ & 5,46 & 6,37 & 1,20 & 13,58 \\
\hline Acido pantoténico & $\mathrm{mg}$ & 0,95 & 1,01 & 0,25 & 2,18 \\
\hline Vitamina B-6 & $\mathrm{mg}$ & 0,30 & 0,34 & 0,04 & 1,30 \\
\hline Folato, total & ug & 38 & 44 & 31 & 79 \\
\hline Vitamina A & IU & 9 & 9 & 0 & 9 \\
\hline Vitamina $\mathrm{E}$ & $\mathrm{mg}$ & 1,01 & 0,82 & 0,05 & 1,49 \\
\hline Vitamina K & ug & 1,90 & 1,90 & 0,30 & 1,90 \\
\hline \multicolumn{6}{|c|}{ Perfil lipidico (ácidos grasos) } \\
\hline Saturados & g & 0,27 & 0,32 & 0,30 & 0,63 \\
\hline Monoinsaturados & g & 0,20 & 0,23 & 0,19 & 0,64 \\
\hline Polinsaturados & $g$ & 0,63 & 0,78 & 0.85 & 2,21 \\
\hline \multicolumn{6}{|l|}{ Carotenoides } \\
\hline Luteina + zeaxantin & na ug & 220 & 220 & 18 & 240 \\
\hline
\end{tabular}

Javier Zuñiga R, 2007. Basado en USDA-ARS 2006 Y National Public Health Institute

Estudios sobre el análisis bromatológico realizado en los laboratorios de La Quisa y laboratorio del departamento de control de calidad de la UNAN-León realizado en la investigación del comportamiento agronómico y económico del trigo jupateco en la Concordia, arrojaron que dicho cultivo tiene los siguientes valores nutricionales: (Tabla 2) 
TABLA 2

Análisis bromatológico

\begin{tabular}{|c|c|c|c|c|c|}
\hline Proteínas & Carbohidratos & Grasa & Ceniza & Humedad & Fibra \\
\hline $10.1 \%$ & $76.7 \%$ & $2.52 \%$ & $1.56 \%$ & $6.22 \%$ & $47.55 \%$ \\
\hline & & & & & \\
\hline
\end{tabular}

Iglesias, D. et al 2012

Dichos datos reflejan el gran aporte de proteínas, carbohidratos y fibra importantes para el desarrollo físico y mental de la humanidad, por tanto, se puede considerar una estrategia nutricional humana y animal, en los planes de desarrollo humano del gobierno de Unidad y Reconciliación Nacional, para aportar a la disminución de la pobreza y el hambre local del departamento de Jinotega, Nicaragua.

\section{Conclusiones}

Nicaragua es un país con una proporción importante de población vulnerable a los cambios climáticos, el $46 \%$ se encuentra en pobreza extrema y más del $25 \%$ de los agricultores producen para su subsistencia; estos pequeños productores no tienen recursos para protegerse de los eventos climáticos y el deterioro ambiental; para ellos, la siembra del cultivo de trigo y la diversificación de sus parcelas, pueden tener grandes impactos en el bienestar de las familias productoras de granos básicos.

Los principales rubros de granos básicos (maíz, arroz y frijol), son de los que depende la economía de Nicaragua y una parte importante del rubro café, por tanto al diversificar un poco más los granos básico introduciendo el rubro del trigo, sería una opción potencial para garantizar la soberanía nutricional del departamento de Jinotega.

Debido a que el trigo tiene una gran variabilidad genética y adaptación climática, podría extenderse a otras zonas sub tropicales de Nicaragua, ayudando a disminuir las importaciones de harina de 36.1 millones de dólares que invierte el estado, así lograr la independencia económica a la cual está apostando el Gobierno de Unidad y Reconciliación Nacional.

Conocer los efectos del cambio climático puede ayudar a los tomadores de decisiones y hacedores de política a tomar decisiones y elaborar políticas agropecuarias acordes a las necesidades de los agricultores, que Los agricultores podrían reducir las pérdidas potenciales del cambio climático incrementando los rendimientos agrícolas en las regiones más afectadas, mediante la aplicación de medidas de adaptación relacionadas con mejores prácticas agrícolas, como por ejemplo:

-Adaptar las fechas de siembra a los nuevos ciclos climáticos, es decir, elegir el momento oportuno de siembra para que las plantas se desarrollen adecuadamente.

Practicar la diversificación de cultivos y seleccionar las variedades que se adapten a las nuevas condiciones climáticas. De la misma forma considerar las condiciones agroecológicas de la región para adoptar los cultivos más adecuados y disminuir el riesgo de pérdida de cosechas y reducir los costos.

Incorporando un modelo sistema de producción agreoecologica del cultivo de trigo en Nicaragua podríamos garantizar la sustentabilidad económica y productiva garantizando la protección del bien agua, tierra y plantas.

En Nicaragua la producción de trigo harinero representaría una oportunidad de independencia económica, ya que se terminó la donación de trigo de Rusia y ahora tendrá que comprar la materia prima a Rusia o Canadá países con los que ha hecho negociaciones de comercialización de trigo. 


\section{Referencias Bibliográficas}

Altieri, M. A., \& Toledo, V. (2011). La revolución agroecológica de América Latina1. Segui buscando en la Red de Bibliotecas Virtuales de CLACSO http://biblioteca. clacso. edu. ar, 163.

Cañarejo, P., \& Adolfo, S. (2013). Evaluación de siete variedades de trigo (Triticum aestivum L.) con tres tipos de manejo nutricional, a $3220 \mathrm{msnm}$ Olmedo Cayambe- 2012. Universidad Politécnica Salesiana. https:// dspace.ups.edu.ec/handle/123456789/6052

Financiera rural (2010). Monografía del trigo grano. (en línea).México. Consultado el 10 may. 2013. Disponible en http://www.financierarural.gob.mx/informacionsectorrural/Documents/Monografias /Monograf\%C3\%ADa \%20Trigo\%20(dic\%2010)\%20vf.pdf.

Iglesias, D, et al 2012. Caracterización agronómica y económica del trigo Jupateco. Tesis para optar el título de ingeniero en agroecología tropical. Universidad Nacional Autónoma de Nicaragua, CUR Jinotega.

Joachim von Braun, M. S. Swaminathan y Mark W. Rosegran. (2003) IFPRI (Instituto internacional de investigación sobre políticas alimentarias)," ensayo Agricultura, seguridad alimentaria, nutrición y los objetivos del milenio". Ensayo reimpreso del informe anual 2003 - $20004 . P p$ 2-4.

Made- in -Argentina. (2010). Caracteristicas del cultivo de trigo. (en línea). Consultado el 10 de jul.2013. Disponible en http://www.made- inargentina.com/alimentos/granos\%20y\%20oleaginosas/temas \%20relacionados/caracteristicas \%20\%20del\%20 cultivo\%20de\%20trigo.htm.

MIFIC (Ministerio de Fomento Industria y Comercio). (2012). Informe Ejecutivo de Comercio Exterior EneroOctubre (en línea). Managua, Nicaragua. Consultado el 16 nov.2013. Disponible en: http://www.mific.gob.ni/ LinkClick.aspx $?$ fileticket $=$ AnMl0fEobf0\%3D\&tabid $=772$ \&language $=$ es-NI.

Miller, T. (2000). Estadios de Crecimiento del cultivo de Trigo. Texas, Estados Unidos. Consultado el 12 de sep.2013. Disponible en: http://www.ipni.net/publication/ialacs.nsf/0/5cle5e74ae5d78aa8525799c0058 efb6/\$file/estadiofeekes.pdf

Moreno. O. (2014). Siembra de trigo en surco para el valle de Mexicali, B.C. Mexico.

Platero, G. G. R., Cortés, A. M. R., Castillo, D. P., Hernández, L. H., \& Ramírez, J. G. S. (2015). Bancos Comunitarios de Semillas Criollas. CATIE

Restrepo, J., Diego Ivan, S., y Prager, M. (2000). Agroecología. Editorial CEDAC. Republica Dominicana.pag.6.

Sánchez, E (2009). Soñadores del trigo. Recuperado en http://www.elnuevodiario.com.ni/especiales/47877sonadores-siembra-trigo-harinero- nicaragua/. 2012/04/10.

Ugarteche, O. Segovia y D. (2010). El trigo otro commodity financiarizado. Mexico. Ed. UNAM, Mexico.pp1-10.

Villaseñor-Espín, Ó. M., Huerta-Espino, J., Leyva Mir, S. G., Villaseñor-Mir, H. E., Singh, R. P., Sandoval-Islas, J. S., \& Espitia-Rangel, E. (2009). Genética de la resistencia a roya amarilla en plantas adultas de trigo harinero. Revista fitotecnia mexicana, 32(3), 217-223.

Watson, O. (2012). En línea Cambio climático. Recuperado en http://cambioclimaticoglobal.com/que-es-el-cambioclimatico.

Zúñiga, Javier, R. (2007). Trigo blanco valor nutricional y potencial. Revista INIA tierra. N 74 